\title{
The descent and bouncing path of the Hayabusa2 lander MASCOT at asteroid (162173) Ryugu
}

\author{
F. Scholten ${ }^{1}$, F. Preusker ${ }^{1}$, S. Elgner ${ }^{1}$, K.-D. Matz ${ }^{1}$, R. Jaumann ${ }^{1}$, J. Biele ${ }^{2}$, D. Hercik ${ }^{3}$, H.-U. Auster ${ }^{3}$, M. Hamm ${ }^{1}$, \\ M. Grott ${ }^{1}$, C. Grimm ${ }^{4}$, T.-M. Ho ${ }^{4}$, A. Koncz ${ }^{1}$, N. Schmitz ${ }^{1}$, F. Trauthan ${ }^{1}$, S. Kameda ${ }^{5}$, S. Sugita ${ }^{6,7}$, R. Honda ${ }^{8}$,

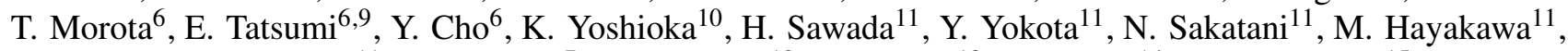 \\ M. Matsuoka ${ }^{11}$, M. Yamada ${ }^{7}$, T. Kouyama ${ }^{12}$, H. Suzuki ${ }^{13}$, C. Honda ${ }^{14}$, and K. Ogawa ${ }^{15}$
}

(Affiliations can be found after the references)

Received 23 September 2019 / Accepted 31 October 2019

\begin{abstract}
Images from the Optical Navigation Camera system (ONC) onboard the Hayabusa2 spacecraft show the MASCOT lander during its descent to the surface of asteroid (162173) Ryugu. We used results from a previous stereo-photogrammetric analysis that provided precise ONC image orientation data (camera position and pointing), ONC orthoimages, and an ONC-based 3D surface model to combine them with the visibilities of MASCOT itself and its shadow on-ground within the ONC images. We integrated additional information from instruments onboard MASCOT (MASMag, MARA, MASCam) and derived MASCOT's release position and modeled its free-fall descent path and its velocity over $350 \mathrm{~s}$ from its release at $\sim 41 \mathrm{~m}$ altitude above ground until its first contact with the surface of Ryugu. After first contact, MASCOT bounced over the surface of Ryugu for $663 \mathrm{~s}$ and came to rest at its first settlement point after four intermediate surface contacts. We again used ONC images that show MASCOT and partly its shadow and reconstructed the bouncing path and the respective velocities of MASCOT. The achieved accuracy for the entire descent and bouncing path is $\sim 0.1 \mathrm{~m}(1 \sigma)$.
\end{abstract}

Key words. minor planets, asteroids: general - planets and satellites: surfaces - minor planets, asteroids: individual: (162173) Ryugu

\section{Introduction}

After its launch in 2014 , the spacecraft of the Japanese Hayabusa2 (HY2) mission (Watanabe et al. 2017) reached its target, asteroid (162173) Ryugu, in 2018 (Watanabe et al. 2019). On 3 October 2018, HY2 performed its descent towards Ryugu for the release of the German-French MASCOT lander (Mobile Asteroid surface SCOuT, Ho et al. 2017) from low altitude. The Optical Navigation Camera system (ONC) onboard the HY2 spacecraft (Kameda et al. 2017) acquired a set of images before, during, and after the release of MASCOT (Sugita et al. 2019), while MASCOT's German camera MASCam (Jaumann et al. 2017, 2019) acquired images during MASCOT's descent, its bouncing phase, and on-ground after coming to rest on the surface of Ryugu. For the full exploitation of the MASCam image data, for example for comparison with ONC orthoimages (Preusker et al. 2019) of the surface of Ryugu, it is necessary to reference the different images geometrically in body-fixed coordinates and to derive their characteristics (e.g., scale, geolocation, information about observation and illumination conditions). Furthermore, for the derivation of physical properties of Ryugu's regolith (e.g., compressive strength), precise knowledge of the impact velocities is required. Finally, mainly as an engineering aspect, but of great importance for the success of future lander missions and valuable for the scientific analysis of the acquired data, investigations of the performance of the release mechanism of MASCOT onboard HY2 can be supported by precise measurements of the effective release velocity. Within this Letter, we refine initial results (Jaumann et al. 2019) and describe how we combined image orientation data with image analysis of MASCOT and its shadow on ground in several ONC images. We reconstruct the position and velocity of MASCOT continuously from its release to the first contact with the surface and during its bouncing path until the first settlement position on Ryugu. Complementary to this work, the analysis of the geometry during on-ground operations is given in Scholten et al. (2019).

\section{Relevant input information for the descent}

For our analysis, we used the SPICE kernel system (Acton 1996) as the standard tool and dataset in planetary science. As the reference frame for the reconstruction of the descent path of MASCOT from its release until its first contact on the surface, we chose a Ryugu body-fixed coordinate frame (RBFF). In order to be consistent with all activities in the context of MASCOTrelated science, we adopted the commonly agreed definition of the pole orientation and zero longitude of the RBFF in the inertial J2000 coordinate frame. The respective SPICE planetary constants kernel as well as improved ONC image orientation data (see Sect. 2.4) are described in detail in Preusker et al. (2019). Together with our results (see Sect. 5), we provide all other relevant SPICE kernels, for example describing the position of the Sun for any given UTC time (required for the interpretation of MASCOT's shadow positions). In the following, we describe other context information that constrains major parameters of MASCOT's descent path.

\subsection{HY2 and MASCOT body-fixed coordinate frames}

MASCOT is a rectangular box with the following dimensions: $0.28 \mathrm{~m} / 0.29 \mathrm{~m} / 0.21 \mathrm{~m}$ (Ho et al. 2017). The reconstruction of the 

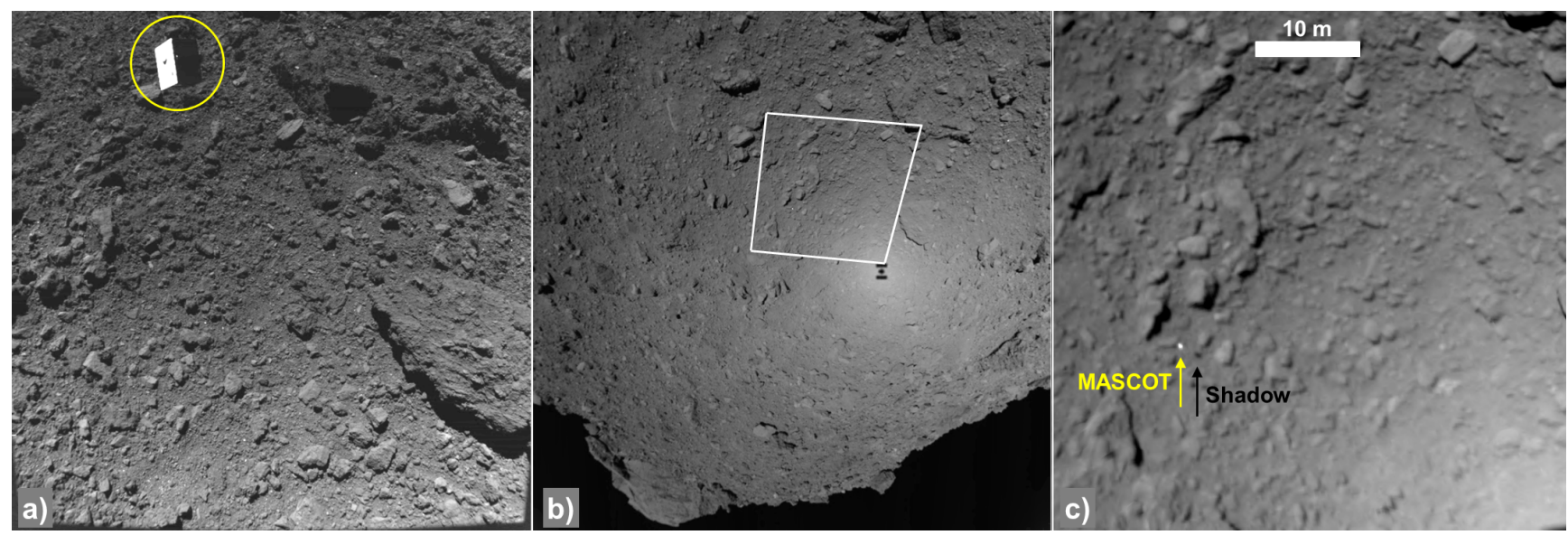

Fig. 1. Examples of ONC images that show MASCOT during descent (stretched for display). Panel a: original ONC-W2 image hyb2_onc_20181003_015814_w2f, 53.8 s after $t_{0}$. Panel b: original ONC-W1 image hyb2_onc_20181003_020252_w1f, $22.4 \mathrm{~s}$ before first ground contact. Panel c: $50 \mathrm{~m} \times 50 \mathrm{~m}$ subset (see white borderline in original image) of the ONC-W1 orthoimage.

descent path of MASCOT is primarily based on MASCOT's visibility in ONC images. MASCOT is resolved to only a few pixels (even with ONC image scales as small as $\sim 5 \mathrm{~cm} \mathrm{pixel}^{-1}$ ). Therefore, path coordinates refer to the geometric center of MASCOT, rather than to the MASCOT body-fixed coordinate frame (MBFF), which is centered on the bottom plate of MASCOT. Thus, for any conversion of derived path coordinates of the geometric center of MASCOT to coordinates of the MBFF origin of MASCOT's center of mass, the respective offset must be taken into account. Until its release, MASCOT was connected to HY2 by the Mechanical and Electronics Support Structure (MESS, Lange et al. 2017). The X/Y/Z coordinates of the origin of the MESS BFF in the HY2 body-fixed frame (HBFF) are $-0.00625 \mathrm{~m} /-0.508 \mathrm{~m} / 0.95793 \mathrm{~m}$ (CNES 2018), with one of the edges of MASCOT located at the origin of the MESS BFF. The MESS structure is titled $15^{\circ}$ downward (in HBFF_z direction). The coordinates of the $\mathrm{ONC}$ (more precisely, the entrance pupils of these cameras to which the positions mentioned in Sect. 2.4 refer) in the HBFF are $0.470 \mathrm{~m} /-0.454 \mathrm{~m} / 0.168 \mathrm{~m}$ for ONC-W1 and $-0.750 \mathrm{~m} /-0.4583 \mathrm{~m} / 0.4838 \mathrm{~m}$ for ONC-W2 (Suzuki et al. 2018).

\subsection{Gravitational acceleration}

The effect of the gravitational acceleration during the descent in low gravity is small and depends on the actual distance to the center of Ryugu. It can be calculated from the GM value of Ryugu of $30.0 \pm 0.4 \mathrm{~m}^{3} \mathrm{~s}^{-2}$ (Watanabe et al. 2019). We considered the respective increase from $g_{\mathrm{R} 485}=0.128 \mathrm{~mm} \mathrm{~s}^{-2}$ at the release position at $485 \mathrm{~m}$ to $g_{\mathrm{R} 450}=0.148 \mathrm{~mm} \mathrm{~s}^{-2}$ at the local radius of $450 \mathrm{~m}$ at the touchdown site (see Sects. 3 and 4). Higher-order gravitational moments at the surface of an irregularly shaped object are far smaller (at the millimeter scale) and can be neglected here. Finally, we assumed for the following calculations that the direction of the gravitational acceleration follows the local ellipsoidal normal vector of the best-fit ellipsoid (Watanabe et al. 2019), that is, $6^{\circ}$ inclined to the north compared to the spherical normal vector.

\subsection{Event times and clock drifts}

Biele \& Fischer (2019) analyzed the different timescales and clock drifts that are relevant for a combined analysis of
HY2/MASCOT-related events. As a consequence, for images during the epoch of MASCOT's descent, ONC image times have to be corrected by $-1.2 \mathrm{~s}$, while MASCam image times have to be corrected by $-451.6 \mathrm{~s}$ in order to tie image times to information given in the coordinated universal time (UTC) frame (e.g., Sun position). From initial analysis of the data acquired by the MASCOT Magnetometer (MASMag), UTC times for two essential events can be extracted, namely the UTC time of the start of MASCOT leaving MESS $\left(t_{0}=2018-10-03\right.$ 01:57:19.16) and, with the MASMag-based duration of $354.69 \mathrm{~s}$ for the descent until the first contact point (CP1) on the surface, we get a first contact time of $t_{\mathrm{CP} 1}=2018-10-03$ 02:03:13.85.

\subsection{ONC context of the MASCOT landing site}

Within a global stereo-photogrammetric block adjustment, Preusker et al. (2019) determined precise orientation data (ONC position and pointing, in inertial frame and in the RBFF) for the majority of ONC images that show the MASCOT landing site at different scales, from meter/pixel image scale during earlier mission periods to centimeters/pixel close before, during, and after the release of MASCOT. Preusker et al. (2019) also derived high-resolution 3D surface models and subsequently ONC orthoimages (images that have been rectified based on the surface model) at the $\sim 5 \mathrm{~cm}$ accuracy level. Both surface models and orthoimages are referenced in the RBFF. The ONC image dataset consists of images from the wide-angle cameras ONC$\mathrm{W} 1 / \mathrm{W} 2$, as well as from the narrow-angle camera ONC-T. We note that there are no ONC-T images that show MASCOT during its descent and bouncing phase. The range of the image scales is $\sim 5-50 \mathrm{~cm} \mathrm{pixel}^{-1}$.

\subsection{Visibility of MASCOT and its shadow on the ground in ONC images}

In total, ten ONC images show MASCOT during its descent. The first two ONC-W2 images show only parts of MASCOT immediately after its release and have not been used in this analysis. The third ONC-W2 image (Fig. 1a) shows MASCOT completely, $\sim 53.8 \mathrm{~s}$ after $t_{0}$, still close to the HY2 spacecraft. The following seven ONC-W1 images show MASCOT and its shadow on the ground during the descent until shortly before first contact on the surface (Figs. 1b and c). 


\section{The descent of MASCOT to the surface of Ryugu}

We used unified UTC time stamps (see Sect. 2.1) for specific events, for example when MASCOT begins to leave MESS onboard HY2, and HY2 spacecraft positions and orientation (from SPG adjustment of ONC images around the release) for the determination of the starting point at $t_{0}$ where MASCOT began to leave MESS. Microgravity separation tests during the development of MASCOT (Grimm et al. 2019) predicted some soft contacts within the MESS guiding structure during the first approximately three seconds of the release. Therefore, the release of MASCOT (MR) and the start of its pure free-fall was set to $t_{\text {start }}=t_{0}+4 \mathrm{~s}=2018-10-03 \quad 01: 57: 23.16$. From Preusker et al. (2019), we obtained ONC positions (of the entrance pupils of the cameras) before and after the release of MASCOT that describe the descent of HY2. We interpolated the position of MASCOT at $t_{0}$ between the two relevant ONC positions and also considered the HBFF offsets between MASCOT and the ONC and retrieved MASCOT's position at $t_{\text {start }}$ as the starting point of the free-fall descent, that is, $25.0891^{\circ}$ south, $319.8039^{\circ}$ east, $484.656 \mathrm{~m}$ radius. The respective distance (release altitude) in radial direction to the surface of Ryugu that is given by the ONC-based surface model is $41.0 \mathrm{~m}$.

Seven ONC-W1 orthoimages (Preusker et al. 2019) show MASCOT and its shadow during the descent. The last images, $\sim 54.4 \mathrm{~s}$ and $\sim 22.4 \mathrm{~s}$ before $t_{\mathrm{CP} 1}$, show MASCOT in $\sim 10 \mathrm{~m}$ and $\sim 6 \mathrm{~m}$ altitude. From extrapolation of their shadow positions, we derived the first contact point on the surface (CP1) at a steep edge of a boulder-like structure of $\sim 3 \mathrm{~m}$ in height. Unfortunately, at the time of the contact, this edge was in shadow and, thus, the ONC surface model is of limited accuracy at this location and the steep edge may even contain an overhang or cavity. Therefore, we could only determine the lateral position of CP1 to the accuracy level of $0.1-0.2 \mathrm{~m}$ in a first step, that is $-22.60^{\circ}$ south, $314.92^{\circ}$ east. The shape model at CP1 shows a radial component of $448.94 \mathrm{~m}$. Due to effects from image matching and interpolation within the generation of the shape model caused by the hidden (shadowed) area at this location, we assumed that the shape model is somewhat smoothened near CP1. Therefore, we selected $450 \mathrm{~m}$ as an initial value for the radius at CP1.

For the reconstruction of the descent path, we reduced the difference of the given coordinates for $t_{\mathrm{start}}$ and $t_{\mathrm{CP} 1}$ by effects from Ryugu's rotation and computed a mean three-dimensional velocity vector $v_{\text {start }}$ as a start value at $t_{\text {start }}$. We then performed a 3D propagation of the descent path while considering gravitational acceleration at particular altitudes during the descent. Within an iterative process, we adjusted $v_{\text {start }}$ until the descent exactly meets the coordinates of CP1. We performed this iteration with different assumptions for the radii at $\mathrm{CP} 1$, varying from $453 \mathrm{~m}$ to $447 \mathrm{~m}$, and retrieved a set of descent paths for the set of $\mathrm{CP} 1$ heights. For the selection of the best-fit descent path from different assumptions for the CP1 height, we made use of the two additional sets of constraints that are given from the visible positions of MASCOT and from its shadows on the ground in seven ONC images. On the one hand, the combination of the adjusted camera orientation data (position and pointing) for these ONC-W1 images, the respective pixel positions of MASCOT within the images, and the geometrical ONC-W1 camera model (focal length and distortion) defines 3D vectors (lines of sight) for the respective image times. The distances between the descent paths at the respective image times and these lines of sight provide accuracy measurements for each solution within the set of descent paths. On the other hand, we defined 3D vectors from the Sun to the shadow positions on the ground for the respective image times. The distances between these vectors and the path positions at the respective image times provide additional accuracy measurements for each descent path. As a result and as the final solution, we found that the best-fit to the criteria (positions of MASCOT and its shadow) can be achieved with a radius of $449.9 \mathrm{~m}$ for $\mathrm{CP} 1$.

The mean deviation between the respective path and the two sets of constraints is $5.2 \mathrm{~cm}$, with a maximum deviation of $11.2 \mathrm{~cm}$. The conclusion from the $\mathrm{CP} 1$ radius value is that CP1 is obviously in the upper part of the boulder, and the shape model is, as expected, degraded $(0.96 \mathrm{~m}$ too low) on this side of the boulder which is in shadow. The final CP1 coordinates are $-22.595^{\circ}$ south, $314.914^{\circ}$ east, $449.9 \mathrm{~m}$ radius. The final velocities of MASCOT at CP1 are $11.8 \mathrm{~cm} \mathrm{~s}^{-1}$ horizontally $\left(10.1 \mathrm{~cm} \mathrm{~s}^{-1}\right.$ to the west, $6.1 \mathrm{~cm} \mathrm{~s}^{-1}$ to the north) and $12.3 \mathrm{~cm} \mathrm{~s}^{-1}$ radially (downward), comprising a total 3D impact velocity of $17.0 \mathrm{~cm} \mathrm{~s}^{-1}$. The RBFF velocities of MASCOT at release are $12.0 \mathrm{~cm} \mathrm{~s}^{-1}$ horizontally $\left(10.7 \mathrm{~cm} \mathrm{~s}^{-1}\right.$ to the west, $5.5 \mathrm{~cm} \mathrm{~s}^{-1}$ to the north) and $7.7 \mathrm{~cm} \mathrm{~s}^{-1}$ radially (downward), comprising a total start velocity in RBFF of $14.2 \mathrm{~cm} \mathrm{~s}^{-1}$. The respective release velocities of MASCOT in HBFF (relative to HY2 at release) are of importance for the analysis of the release mechanism; they are $-0.15 \mathrm{~cm} \mathrm{~s}^{-1}$ in HBFF_x, $-5.73 \mathrm{~cm} \mathrm{~s}^{-1}$ in HBFF_y, and $-1.28 \mathrm{~cm} \mathrm{~s}^{-1}$ in HBFF_z direction, comprising a total 3D release velocity relative to $\mathrm{HY} 2$ of $5.9 \mathrm{~cm} \mathrm{~s}^{-1}$. The respective downward tilt in the HY2 frame is $12.6^{\circ}$, close to the nominal value of $15^{\circ}$ (compare with Sect. 2.1). We present the results for the descent phase (RBFF coordinates and velocities during descent) in graphical (Fig. 2) and tabular form (first part of Table A.1). At the time of the ONC-W2 image shortly after the release (Fig. 1a), the distance of the entrance pupil of ONC-W2 to MASCOT is $3.25 \mathrm{~m}$.

\section{The bouncing of MASCOT over the surface of Ryugu}

After its first contact on the surface of Ryugu at CP1, MASCOT is visible in 12 ONC-W1 context images during its bouncing phase. The last of these ONC-W1 images was taken at $02: 14: 03.52$, immediately before the end of the bouncing phase (the MARA radiometer determined an at-rest-status at $02: 14: 17 \pm 30 \mathrm{~s})$. After a final sliding on the surface, MASCOT reached its first settlement point (SP1) at 02:18:51 (Jaumann et al. 2019). More precisely, sensors of the Guidance, Navigation and Control system (GNC; Ho et al. 2017) detected that there had not been a change in MASCOT's position for about one minute before 02:18:51. Below, we show that this final sliding is very small ( $\leqq 1 \mathrm{~cm}$, see Sect. 4.4). Therefore, we define the arrival time at SP1 as 02:14:17. From MASMag measurements, CP1 appears to be a multiple-contact event that lasted $\sim 7.25 \mathrm{~s}$ until $\mathrm{CP} 2$, which is the starting point of a first free-fall bounce. MASMag also recorded three surface contacts, CP3/4/5. Therefore, we considered the first free-fall bounce from CP2 to CP3 (119.5 s), a second bounce from CP3 to CP4 (173.5 s), a third bounce from CP4 to CP5 (136.4 s), and a fourth bounce from CP5 to SP1 $(226.5 \mathrm{~s})$. The first four ONC-W1 images clearly show MASCOT's shadow on the ground during the first bounce, while the fifth image and all subsequent ONC-W1 images during the following bounces do not show the shadow. Because of these variable constellations, we modeled the four bounces with different approaches. We present the final results for the bouncing phase as RBFF coordinates and velocities in graphical form (Fig. 3) and in tabular form (second part of Table A.1). 

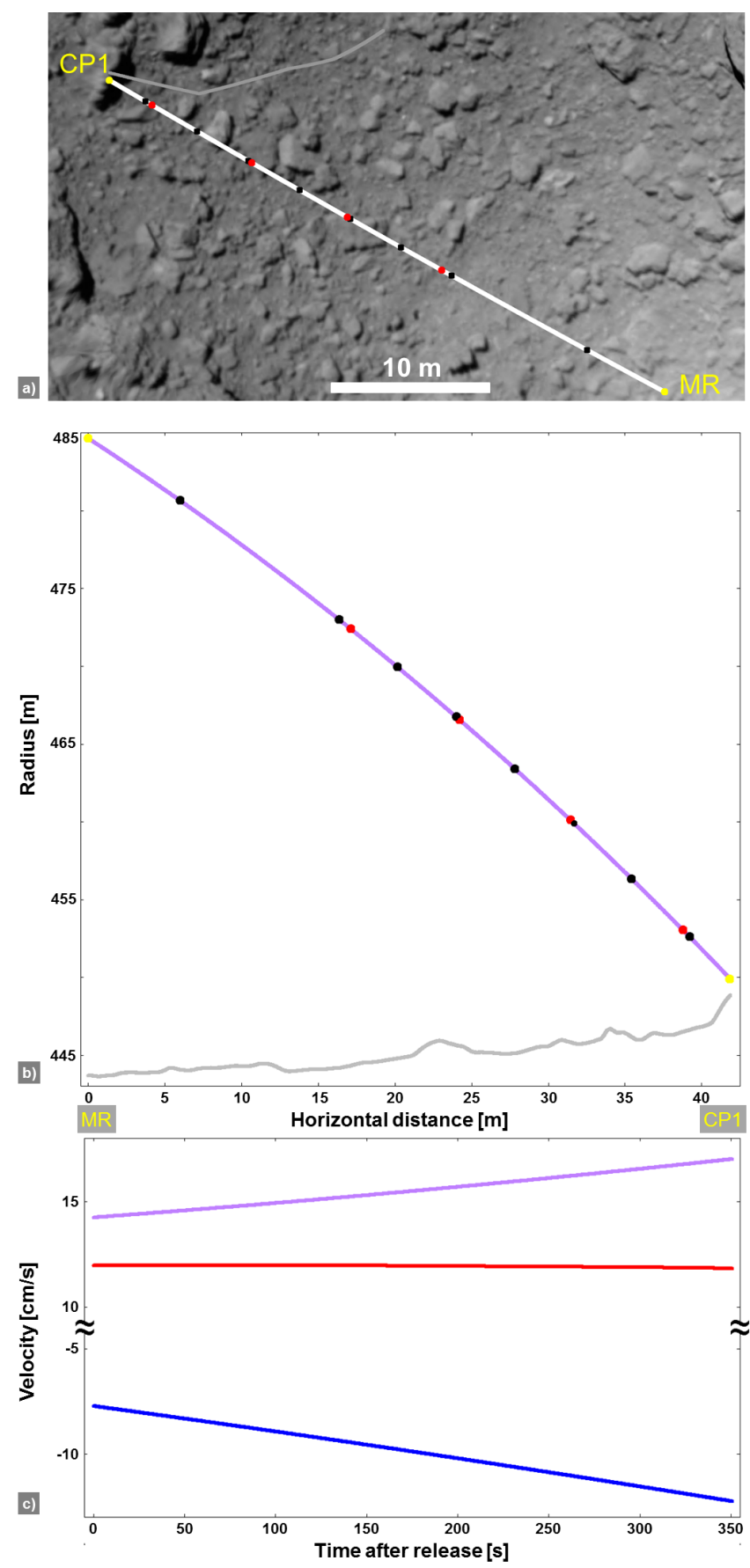

Fig. 2. Descent of MASCOT to the surface of Ryugu from release (MR) to its first contact on the ground (CP1) with events as dots in panels a and $b$. Yellow dots: release and first ground contact, black dots: position of MASCOT when seen in ONC images, red dots: position of MASCOT at the time of four MASCam images. Panel a: ground profile of MASCOT's descent path on top of ONC orthoimage map (thin line: MASCOT's bouncing path). Panel $b$ : vertical profile of MASCOT's descent path (purple) and the surface of Ryugu (gray). Panel c: MASCOT's descent velocities over time (blue: vertical, red: horizontal, purple: total 3D).

\subsection{First bounce of MASCOT from CP2 to CP3}

Similar to the reconstruction of the descent path, we used the 3D line-of-sight vectors from the ONC-W1 camera positions to MASCOT itself and the $3 \mathrm{D}$ vectors from the Sun to the shadow positions for the determination of the four MASCOT
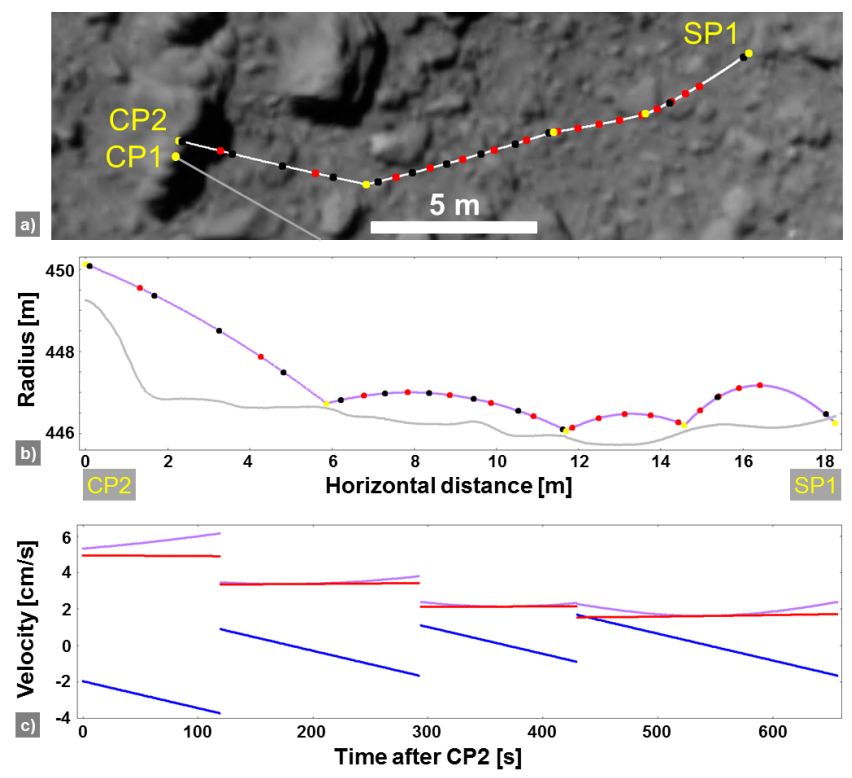

Fig. 3. Bouncing phase of MASCOT from second contact (CP2) to its first settlement point (SP1) with events as dots in panels $a$ and $b$. Yellow dots: positions of contact points, black dots: position of MASCOT when seen in ONC images, red dots: position of MASCOT at the time of sixteen MASCam images. Panel a: ground profile of MASCOT's bouncing path on top of ONC orthoimage map (thin line: MASCOT's descent path). Panel $b$ : vertical profile of MASCOT's bouncing path (purple) and the surface of Ryugu (gray). Panel $c$ : bouncing velocities of MASCOT over time (blue: vertical, red: horizontal, purple: total 3D).

positions within this first bounce (ONC images at 02:03:23, 02:03:55, 02:04:27, 02:04:59). As for the reconstruction of the descent, the shadow positions are known from ONC orthoimages. For each ONC image, we defined the initial coordinates of MASCOT in the center of the closest distance between the respective two rays. The mean distance between these MASCOT positions and the two rays is $8.3 \mathrm{~cm}$, with a maximum distance of $13.0 \mathrm{~cm}$, which is of a similar accuracy to that of the reconstruction of the descent path, and is intitial proof of the accuracy of the reconstruction of the descent. Finally, using these four MASCOT positions as constraints, we determined a best-fit free-fall model for the first bounce using $g_{\mathrm{R} 450}$ (see Sect. 2.2). This model yields the coordinates of the first bouncing path, including CP2 and CP3. The mean deviation of this best-fit model from the four measured MASCOT positions is $5.0 \mathrm{~cm}$, with a maximum deviation of $9.1 \mathrm{~cm}$, an indication of the achieved accuracies. The 3D distance between CP1 and CP2 (i.e., the total movement of MASCOT during the multiple-contact event at CP1) is $0.54 \mathrm{~m}$, mainly northwards from CP1 (see Fig. 3). As further proof of the accuracy of our results, the radius vector of $446.73 \mathrm{~m}$ of the modeled CP3 position is just $0.11 \mathrm{~m}$ higher than the ONC surface model radius at $\mathrm{CP} 3$, a difference which is highly plausible for a surface contact given the dimensions of MASCOT and the fact that the modeled path refers to the center of the MASCOT lander. The 3D distance between CP2 and CP3 is $6.76 \mathrm{~m}(5.84 \mathrm{~m}$ horizontal, $3.40 \mathrm{~m}$ vertical).

\subsection{Second bounce of MASCOT from CP3 to CP4}

MASCOT is visible in six ONC-W1 images (02:05:31, 02:06:03, 02:06:35, 02:07:07, 02:07:39, 02:08:11), and no shadows are visible (completely or at least partly obscured by MASCOT itself) after CP3. Therefore, we started the modeling of this 
second bounce from the previously derived CP3 position and used the MASCOT positions from the ONC orthoimages as constraints. We corrected these positions because of displacements from MASCOT's altitude above the surface that we initially assumed to range from a few decimeters up to $1 \mathrm{~m}$. Higher altitudes are not likely because of the obscured shadow. As an additional constraint, we used the last MASCOT position and its surface radius value from the ONC-based surface model as an approximation for $\mathrm{CP} 4$, since the last $\mathrm{ONC}$ image was acquired just $2.6 \mathrm{~s}$ before $\mathrm{CP} 4$. With an iterative process (including iteratively improved displacement corrections from modeled altitudes above ground), we retrieved the best-fit free-fall model for the second bouncing path up to CP4. The mean deviation of this best-fit model from the six measured MASCOT positions is $7.7 \mathrm{~cm}$, with a maximum deviation of $18.3 \mathrm{~cm}$. The slightly higher deviations result from the increasing pixel scale of the ONC images (acquired during the ascent of $\mathrm{HY} 2$ after the release of MASCOT). The pixel scales of the six ONC images increase from 26 to $36 \mathrm{~cm} \mathrm{pixel}^{-1}$. Thus, the achieved accuracy is still well within one ONC image pixel. The 3D distance between CP3 and CP4 is $5.88 \mathrm{~m}$ (5.84 m horizontal, $0.67 \mathrm{~m}$ vertical).

\subsection{Third bounce of MASCOT from CP4 to CP5}

There are no ONC images that show MASCOT during this bounce. However, with the previously derived CP4 coordinates, the coordinates of CP5 from the modeling procedure for the fourth bounce (see Sect. 4.4), the known time between CP4 and $\mathrm{CP} 5$, and the given gravitational acceleration, it was possible to determine the start velocity and the entire third bouncing path directly. Since this bounce is not independently controlled by additional ONC observations, its accuracy depends on the accuracy of the CP4 and CP5 coordinates and is therefore expected to be similar to the fourth bounce. The 3D distance between $\mathrm{CP} 4$ and CP5 is $2.90 \mathrm{~m}$ ( $2.89 \mathrm{~m}$ horizontal, $0.15 \mathrm{~m}$ vertical $)$.

\subsection{Fourth bounce of MASCOT from CP5 to SP1}

MASCOT is visible in two ONC-W1 images during this fourth bounce (02:11:23, 02:14:03). For the second one, which shows MASCOT just $14 \mathrm{~s}$ before coming to rest on the surface of Ryugu, we used the respective radius value from the surface model (with $30 \mathrm{~cm}$ add-on for its assumed height above ground) together with its lateral position from the orthoimage as fixed coordinates for this MASCOT position. The measured MASCOT position at 02:11:23 was used as a second constraint for the free-fall model. A third constraint tied the radius of the start point CP5 of this fourth bounce to the respective radius in the surface model. With these constraints, we finally derived the start velocity and the position of CP5. Arrival and departure velocities at CP5 (see Table A.1) indicate a significantly smaller relative loss of velocity of MASCOT on a boulder-like structure than at previous ground contacts. Fourteen seconds after the second ONC image, the retrieved free-fall model for the fourth bounce ends at 02:14:17 at MASCOT's first settlement point (SP1), right at the edge of a boulder of $\sim 1 \mathrm{~m}$ in size. This is the reason why, similar to $\mathrm{CP} 1$, the surface model is slightly degraded, $\sim 30 \mathrm{~cm}$ too high (see Fig. 3). Two MASCam images were taken at SP1. These images are mostly dark and show only very faint structures, which indicates that MASCOT rested on its $+y$-side with MASCam facing directly toward the surface. The structures show that MASCOT's final movement at SP1 is very small $(<1 \mathrm{~cm})$. The total 3D distance of this fourth and last bounce from CP5 to $\mathrm{SP} 1$ is $3.67 \mathrm{~m}$ ( $3.67 \mathrm{~m}$ horizontal, $0.04 \mathrm{~m}$ vertical). Due to the increasing altitude during the ascent of the HY2 spacecraft, the resolution of the last two ONC-W1 images (42 and $49 \mathrm{~cm} \mathrm{pixel}^{-1}$ ) is slightly lower compared to the previous ONC images. Therefore, the uncertainty of the reconstruction of the last part of the bouncing phase (after $\mathrm{CP} 4$ ) is somewhat larger and may be approximately $0.2 \mathrm{~m}$.

\section{Summary}

We combined information from several instruments onboard the MASCOT lander with context information from the ONC onboard the Hayabusa2 spacecraft in order to reconstruct the descent and bouncing path of MASCOT in the Ryugu body-fixed frame over a total time of $17 \mathrm{~min}$ from MASCOT's release until its first settlement point on the surface of Ryugu. In particular, visibilities of MASCOT itself and of its shadow on the ground in ONC images constrained the analysis, leading to a 3D accuracy for the reconstructed path of MASCOT at the $0.1 \mathrm{~m}$ level $(1 \sigma)$ and MASCOT velocities at least at the millimeters per second accuracy level. Due to decreasing image resolution of the last ONC-W1 images, the reconstruction of the last part of the bouncing phase may be slightly less accurate, up to the $0.2 \mathrm{~m}$ level. A full $1 \mathrm{~Hz}$ discretization of MASCOT's entire descent and bouncing path in Ryugu body-fixed frame coordinates and velocities is available at the Europlanet website ${ }^{1}$ together with all other relevant SPICE kernels. The derived information will support forthcoming analyses of the image content of MASCam images and of data from other instruments on the MASCOT lander acquired during descent and bouncing in order to determine some of Ryugu's physical properties and to support geological interpretation of the surface of Ryugu (e.g., from different loss of velocity (energy) of MASCOT at the different contact points). Finally, engineering aspects of the release mechanism may also benefit from the results.

Acknowledgements. The Hayabusa 2 mission is operated by JAXA. The MASCOT lander on the Hayabusa2 mission is a DLR/CNES cooperation. The authors acknowledge funding by JAXA, CNES, DLR, and the work of the different instrument teams of Hayabusa2 and MASCOT that made the different instrument measurements possible. We thank Katharina Otto and Volker Mertens (DLR) for their support during the review and submission process.

\section{References}

Acton, C. H. 1996, Planet. Space Sci., 44, 65

Biele, J., \& Fischer, H. H. 2019, MASCOT-internal Technical Note MCS-TN1053_TimeScales, version 1.1

CNES 2018, MASCOT-internal Technical Note MSC-TN-MA-0078-CNES, version 1.1

Grimm, C., Lange, C., Lange, M., et al. 2019, CEAS Space J., submitted

Ho, T.-M., Baturkin, V., Grimm, C., et al. 2017, Space Sci. Rev., 208, 339

Jaumann, R., Schmitz, N., Koncz, A., et al. 2017, Space Sci. Rev., 208, 375

Jaumann, R., Schmitz, N., Ho, T.-M., et al. 2019, Science, 365, 817

Kameda, S., Suzuki, H., Takamatsu, T., et al. 2017, Space Sci. Rev., 208, 17

Lange, M., Hühne, C., Lange, C., \& Mierheim, O. 2017, 68th International Astronautical Congress (IAC) No. C2.7.9 (Adelaide, Australia: IAF)

Preusker, F., Scholten, F., Elgner, S., et al. 2019, A\&A, 632, L4

Scholten, F., Preusker, F., Elgner, S., et al. 2019, A\&A, 632, L5

Sugita, S., Honda, R., Morota, T., et al. 2019, Science, 364, eaaw0422

Suzuki, H., Yamada, M., Kouyama, T., et al. 2018, Icarus, 300, 341

Watanabe, S., Tsuda, Y., Yoshikawa, M., et al. 2017, Space Sci. Rev., 208, 3

Watanabe, S., Hirabayashi, M., Hirata, N., et al. 2019, Science, 364, 268

http://europlanet.dlr.de/MASCOT 
${ }^{1}$ German Aerospace Center (DLR), Institute of Planetary Research, 12489 Berlin, Germany e-mail: Frank.Scholten@dlr.de

2 German Aerospace Center (DLR), Microgravity User Support Center (MUSC), Cologne, Germany

3 Technical University Braunschweig, Institute for Geophysics and Extraterrestrial Physics, Germany

${ }^{4}$ German Aerospace Center (DLR), Institute of Space Systems, Bremen, Germany

5 Department of Physics, Rikkyo University, 3-34-1 Nishi-Ikebukuro, Toshima, Tokyo, Japan

${ }^{6}$ University of Tokyo, 7-3-1 Hongo, Bunkyo, Tokyo, Japan

7 Planetary Exploration Research Center, Chiba Institute of Technology, 2-17-1 Tsudanuma, Narashino, Chiba, Japan
${ }^{8}$ Department of Information Science, Kochi University, 2-5-1 Akebono, Kochi, Japan

${ }^{9}$ Instituto de Astrofisica de Canarias, University of La Laguna, Santa Cruz de Tenerife, Spain

10 Department of Complexity Science and Engineering, University of Tokyo, 5-1-5 Kashiwanoha, Kashiwa, Chiba, Japan

11 ISAS, JAXA, 3-3-1 Yoshinodai, Chuo, Sagamihara, Kanagawa, Japan

12 National Institute of Advanced Industrial Science and Technology, 2-3-26 Aomi, Koto, Tokyo, Japan

13 Department of Physics, Meiji University, 1-1-1 Higashimita, Tama, Kawasaki, Kanagawa, Japan

14 CAIST/ARC-Space, University of Aizu, Aizu-Wakamatsu, Fukushima, Japan

15 Department of Planetology, Kobe University, 1-1 Rokkodaicho, Nada, Kobe, Hyogo, Japan 


\section{Appendix A: Table}

Table A.1. Times, positions, and velocities for all of MASCOT's descent and bouncing events in RBFF (MCA = MASCam image).

\begin{tabular}{|c|c|c|c|c|c|c|c|c|c|c|c|}
\hline $\begin{array}{l}\text { Event } \\
\text { type }\end{array}$ & $\begin{array}{c}\text { Image } \\
\text { identifier }\end{array}$ & $\begin{array}{c}\text { UTC } \\
2018-10-03\end{array}$ & $\begin{array}{c}\mathrm{X} \\
{[\mathrm{m}]}\end{array}$ & $\begin{array}{c}\mathrm{Y} \\
{[\mathrm{m}]}\end{array}$ & $\begin{array}{c}\mathrm{Z} \\
{[\mathrm{m}]}\end{array}$ & $\begin{array}{l}\text { Latitude } \\
{\left[{ }^{\circ}\right] \text { South }}\end{array}$ & $\begin{array}{c}\text { Longitude } \\
{\left[{ }^{\circ}\right] \text { East }}\end{array}$ & $\begin{array}{c}\text { Radius } \\
{[\mathrm{m}]}\end{array}$ & $\begin{array}{c}v_{\text {hori }} \\
{\left[\mathrm{cm} \mathrm{s}^{-1}\right]}\end{array}$ & $\begin{array}{c}v_{\text {vert }} \\
{\left[\mathrm{cm} \mathrm{s}^{-1}\right]}\end{array}$ & $\begin{array}{c}v_{3 \mathrm{D}} \\
{\left[\mathrm{cm} \mathrm{s}^{-1}\right]}\end{array}$ \\
\hline \multicolumn{12}{|c|}{ Descent } \\
\hline MR & - & $01: 57: 23.2$ & 335.294 & -283.306 & -205.497 & 25.0864 & 319.8039 & 484.679 & 11.98 & -7.69 & 14.23 \\
\hline ONC-W2 & 53161 & 01:58:13.0 & 329.981 & -285.741 & -201.329 & 24.7606 & 319.1097 & 480.696 & 11.98 & -8.30 & 14.57 \\
\hline ONC-W1 & 53164 & 01:59:39.5 & 320.237 & -289.312 & -193.714 & 24.1734 & 317.9044 & 473.052 & 11.97 & -9.39 & 15.21 \\
\hline MCA & 100 & 01:59:46.0 & 319.478 & -289.545 & -193.123 & 24.1281 & 317.8138 & 472.439 & 11.96 & -9.47 & 15.26 \\
\hline ONC-W1 & 53167 & 02:00:11.5 & 316.464 & -290.410 & -190.777 & 23.9491 & 317.4583 & 469.982 & 11.95 & -9.80 & 15.46 \\
\hline ONC-W1 & 53170 & 02:00:43.5 & 312.601 & -291.382 & -187.776 & 23.7208 & 317.0120 & 466.779 & 11.94 & -10.22 & 15.72 \\
\hline MCA & 101 & 02:00:45.4 & 312.369 & -291.436 & -187.596 & 23.7071 & 316.9855 & 466.585 & 11.94 & -10.24 & 15.73 \\
\hline ONC-W1 & 53173 & $02: 01: 15.5$ & 308.648 & -292.225 & -184.712 & 23.4886 & 316.5656 & 463.441 & 11.92 & -10.64 & 15.98 \\
\hline MCA & 102 & 02:01:46.0 & 304.798 & -292.904 & -181.734 & 23.2636 & 316.1400 & 460.132 & 11.90 & -11.05 & 16.24 \\
\hline ONC-W1 & 53176 & 02:01:47.5 & 304.606 & -292.934 & -181.587 & 23.2524 & 316.1191 & 459.966 & 11.90 & -11.08 & 16.26 \\
\hline ONC-W1 & 53179 & $02: 02: 19.5$ & 300.476 & -293.505 & -178.400 & 23.0121 & 315.6724 & 456.353 & 11.88 & -11.51 & 16.54 \\
\hline MCA & 103 & 02:02:47.6 & 296.777 & -293.890 & -175.553 & 22.7978 & 315.2800 & 453.064 & 11.86 & -11.90 & 16.80 \\
\hline ONC-W1 & 53182 & $02: 02: 51.5$ & 296.258 & -293.935 & -175.154 & 22.7678 & 315.2255 & 452.599 & 11.85 & -11.95 & 16.83 \\
\hline CP1 & - & 02:03:13.9 & 293.267 & -294.149 & -172.858 & 22.5950 & 314.9140 & 449.899 & 11.83 & -12.26 & 17.04 \\
\hline \multicolumn{12}{|c|}{ Bouncing } \\
\hline $\mathrm{CP} 2$ & - & $02: 03: 21.1$ & 293.518 & -294.462 & -172.490 & 22.5322 & 314.9080 & 450.126 & 4.93 & -1.96 & 5.31 \\
\hline ONC-W1 & 53185 & $02: 03: 23.5$ & 293.561 & -294.341 & -172.499 & 22.5361 & 314.9240 & 450.078 & 4.93 & -2.00 & 5.32 \\
\hline MCA & 104 & 02:03:48.0 & 293.955 & -293.085 & -172.575 & 22.5747 & 315.0849 & 449.544 & 4.92 & -2.36 & 5.46 \\
\hline ONC-W1 & 53188 & 02:03:55.5 & 294.064 & -292.689 & -172.589 & 22.5863 & 315.1343 & 449.363 & 4.92 & -2.47 & 5.51 \\
\hline ONC-W1 & 53191 & $02: 04: 27.5$ & 294.466 & -290.943 & -172.605 & 22.6345 & 315.3448 & 448.498 & 4.91 & -2.94 & 5.72 \\
\hline $\mathrm{MCA}$ & 105 & 02:04:47.8 & 294.668 & -289.787 & -172.576 & 22.6640 & 315.4785 & 447.871 & 4.90 & -3.24 & 5.87 \\
\hline ONC-W1 & 53194 & 02:04:59.5 & 294.765 & -289.104 & -172.546 & 22.6806 & 315.5554 & 447.481 & 4.90 & -3.41 & 5.97 \\
\hline $\mathrm{CP} 3$ & - & $02: 05: 20.6$ & 294.904 & -287.843 & -172.467 & 22.7100 & 315.6942 & 446.729 & 4.89 & -3.72 & 6.14 \\
\hline CP3 & - & $02: 05: 20.6$ & 294.904 & -287.843 & -172.467 & 22.7100 & 315.6942 & 446.729 & 3.34 & 0.89 & 3.46 \\
\hline ONC-W1 & 53197 & $02: 05: 31.5$ & 295.231 & -287.670 & -172.425 & 22.6994 & 315.7432 & 446.817 & 3.35 & 0.73 & 3.43 \\
\hline MCA & 106 & $02: 05: 48.0$ & 295.706 & -287.386 & -172.345 & 22.6829 & 315.8174 & 446.918 & 3.35 & 0.49 & 3.39 \\
\hline ONC-W1 & 53200 & 02:06:03.5 & 296.128 & -287.097 & -172.252 & 22.6669 & 315.8871 & 446.976 & 3.36 & 0.26 & 3.37 \\
\hline MCA & 107 & $02: 06: 19.6$ & 296.542 & -286.774 & -172.138 & 22.6498 & 315.9594 & 446.999 & 3.37 & 0.02 & 3.37 \\
\hline ONC-W1 & 53203 & 02:06:35.5 & 296.927 & -286.430 & -172.007 & 22.6324 & 316.0308 & 446.984 & 3.37 & -0.21 & 3.38 \\
\hline $\mathrm{MCA}$ & 108 & 02:06:50.0 & 297.257 & -286.097 & -171.872 & 22.6161 & 316.0959 & 446.938 & 3.38 & -0.43 & 3.40 \\
\hline ONC-W1 & 53206 & 02:07:07.5 & 297.628 & -285.669 & -171.689 & 22.5959 & 316.1745 & 446.841 & 3.39 & -0.68 & 3.45 \\
\hline MCA & 109 & 02:07:19.6 & 297.867 & -285.357 & -171.550 & 22.5816 & 316.2288 & 446.747 & 3.39 & -0.86 & 3.50 \\
\hline ONC-W1 & 53209 & 02:07:39.5 & 298.230 & -284.815 & -171.299 & 22.5574 & 316.3181 & 446.547 & 3.40 & -1.15 & 3.59 \\
\hline $\mathrm{MCA}$ & 110 & 02:07:50.0 & 298.406 & -284.515 & -171.156 & 22.5443 & 316.3652 & 446.418 & 3.40 & -1.31 & 3.65 \\
\hline ONC-W1 & 53212 & 02:08:11.5 & 298.733 & -283.868 & -170.837 & 22.5168 & 316.4616 & 446.103 & 3.41 & -1.62 & 3.78 \\
\hline $\mathrm{CP} 4$ & - & 02:08:14.1 & 298.772 & -283.784 & -170.796 & 22.5134 & 316.4738 & 446.060 & 3.42 & -1.66 & 3.80 \\
\hline CP4 & - & 02:08:14.1 & 298.772 & -283.784 & -170.796 & 22.5134 & 316.4738 & 446.060 & 2.11 & 1.11 & 2.39 \\
\hline MCA & 111 & 02:08:21.8 & 298.943 & -283.726 & -170.807 & 22.5105 & 316.4960 & 446.141 & 2.11 & 1.00 & 2.34 \\
\hline MCA & 112 & 02:08:52.0 & 299.558 & -283.447 & -170.807 & 22.4980 & 316.5829 & 446.377 & 2.12 & 0.56 & 2.19 \\
\hline MCA & 113 & 02:09:21.6 & 300.077 & -283.093 & -170.744 & 22.4840 & 316.6681 & 446.477 & 2.13 & 0.12 & 2.13 \\
\hline MCA & 114 & $02: 09: 52.0$ & 300.523 & -282.648 & -170.615 & 22.4678 & 316.7556 & 446.445 & 2.14 & -0.33 & 2.16 \\
\hline MCA & 115 & $02: 10: 23.6$ & 300.892 & -282.097 & -170.412 & 22.4490 & 316.8466 & 446.268 & 2.15 & -0.79 & 2.29 \\
\hline CP5 & - & $02: 10: 30.5$ & 300.961 & -281.964 & -170.359 & 22.4446 & 316.8665 & 446.210 & 2.15 & -0.89 & 2.33 \\
\hline CP5 & - & $02: 10: 30.5$ & 300.961 & -281.964 & -170.359 & 22.4446 & 316.8665 & 446.210 & 1.53 & 1.69 & 2.28 \\
\hline MCA & 116 & 02:10:53.2 & 301.469 & -281.993 & -170.350 & 22.4245 & 316.9118 & 446.568 & 1.55 & 1.34 & 2.04 \\
\hline ONC-W1 & 53242 & $02: 11: 23.5$ & 302.024 & -281.959 & -170.284 & 22.3980 & 316.9678 & 446.896 & 1.57 & 0.91 & 1.81 \\
\hline MCA & 117 & $02: 11: 23.6$ & 302.043 & -281.956 & -170.281 & 22.3970 & 316.9699 & 446.906 & 1.57 & 0.89 & 1.80 \\
\hline MCA & 118 & $02: 11: 54.0$ & 302.529 & -281.837 & -170.146 & 22.3677 & 317.0280 & 447.108 & 1.60 & 0.44 & 1.65 \\
\hline MCA & 119 & $02: 12: 25.6$ & 302.941 & -281.625 & -169.937 & 22.3352 & 317.0884 & 447.174 & 1.62 & -0.02 & 1.62 \\
\hline ONC-W1 & 53248 & $02: 14: 03.5$ & 303.605 & -280.422 & -168.861 & 22.2236 & 317.2732 & 446.460 & 1.70 & -1.45 & 2.23 \\
\hline SP1 & - & $02: 14: 17.0$ & 303.626 & -280.186 & -168.658 & 22.2065 & 317.2991 & 446.249 & 1.71 & -1.65 & 2.38 \\
\hline
\end{tabular}

